\title{
A Pilot Study Assessing the Possible Combined Effect of Physical Activity and PNPLA3 rs738409 Polymorphism on the Risk for Non-Alcoholic Fatty Liver Disease in the Japanese Elderly General Population
}

This article was published in the following Dove Press journal: Diabetes, Metabolic Syndrome and Obesity: Targets and Therapy

\author{
Narumi Muto ${ }^{1, *}$ \\ Kentaro Oniki ${ }^{1} *$ \\ Miku Kudo' \\ Yui Obata' \\ Yuki Sakamoto' \\ Naoto Tokumaru' \\ Tomoko Izuka' \\ Takehisa Watanabe ${ }^{2}$ \\ Koji Otake ${ }^{3}$ \\ Yasuhiro Ogata ${ }^{3}$ \\ Junji Saruwatari ${ }^{1}$ \\ 'Division of Pharmacology and \\ Therapeutics, Graduate School of \\ Pharmaceutical Sciences, Kumamoto \\ University, Kumamoto, Japan; \\ ${ }^{2}$ Departments of Gastroenterology and \\ Hepatology, Faculty of Life Sciences, \\ Kumamoto University, Kumamoto, Japan; \\ ${ }^{3}$ Japanese Red Cross Kumamoto Health \\ Care Center, Kumamoto, Japan \\ *These authors contributed equally to \\ this work
}

Correspondence: Junji Saruwatari Division of Pharmacology and Therapeutics, Graduate School of Pharmaceutical Sciences, Kumamoto University, 5-I Oe-honmachi, Chuo-ku, Kumamoto 862-0973, Japan

$\mathrm{Tel} / \mathrm{Fax}+8$ I 96-37l-45I2

Email junsaru@gpo.kumamoto-u.ac.jp
Purpose: The patatin-like phospholipase domain containing protein 3 (PNPLA3) rs738409 polymorphism (c.444C $>\mathrm{G})$ is the most well-known genetic risk factor for non-alcoholic fatty liver disease (NAFLD), but whether or not physical activity influences the association between the PNPLA3 genotype and risk of NAFLD is unclear.

Patients and Methods: A retrospective longitudinal analysis was conducted among 352 Japanese subjects. Each type of physical activity was assigned a metabolic equivalent (MET), and the subjects were classified into sedentary, low or high groups using the "METS*T" (METs $\times$ hours per week) value of 5 or 21 as a threshold.

Results: Among the PNPLA3 G/G genotype carriers, the high and low METS*T groups had a lower risk of NAFLD than the sedentary METS*T group (odds ratio [95\% confidence interval]: 0.14 [0.02-0.99] and 0.16 [0.03-1.04], respectively). Furthermore, the PNPLA3 C/C or C/G genotype carriers showed no significant difference in the risk of NAFLD among the three METS*T groups.

Conclusion: The PNPLA3 rs738409 genotype may be associated with the beneficial effects of physical activity on the risk of NAFLD among elderly Japanese individuals. Further comprehensive investigations are therefore needed to verify the preliminary results.

Keywords: weight status, liver steatosis, patatin-like phospholipase domain containing protein 3, genetic polymorphism

\section{Introduction}

Non-alcoholic fatty liver disease (NAFLD) is one of the most common liver diseases and considered a phenotype of metabolic syndrome in the liver. ${ }^{1-3}$ NAFLD is associated with not only a risk of advanced liver disease (e.g. nonalcoholic steatohepatitis, hepatic cirrhosis and liver cancer) but also metabolic diseases, including type 2 diabetes and cardiovascular disease (CVD). ${ }^{2-5}$ NAFLD patients are frequently obese/overweight and/or suffer from type 2 diabetes, and insulin resistance is a key pathogenic trigger. ${ }^{1-3}$ In addition, aging is an important factor affecting the association between obesity, NAFLD and these-related complications, ${ }^{6,7}$ and early interventions are required to effectively manage correctable metabolic risk factors (e.g. sedentary lifestyle) in elderly populations. ${ }^{7}$ 
Unhealthy dietary patterns and a sedentary lifestyle play a role in NAFLD development. ${ }^{3}$ A communitybased cross-sectional study indicated that regular exercise was associated with a reduced risk of NAFLD. ${ }^{8}$ The primary approach to treating NAFLD is the control of underlying risk factors (e.g. diabetes, hyperlipidemia, obesity and other comorbidities), and lifestyle modification through physical activity and dietary habits is a wellestablished therapeutic strategy. ${ }^{9}$ A recent systemic review indicated a possible relationship between physical activity, especially in resistance exercise, and lipid metabolism in the liver. ${ }^{10}$ However, clinical evidence has shown the existence of individual differences in the beneficial effect of physical activity on the development and progression of NAFLD. ${ }^{9,10}$ Since NAFLD development is attributed to not only behavioral but also genetic factors, ${ }^{1-3}$ physical activity may affect the susceptibility to NAFLD by interacting with genetic risk factors, although the details remain unclear.

The patatin-like phospholipase domain containing protein 3 (PNPLA3) rs738409 polymorphism (encoding I148M) has been consistently recognized to be a major genetic risk factor for the development and progression of NAFLD and advanced liver diseases, including steatohepatitis, cirrhosis and liver cancer. ${ }^{3,11,12}$ PNPLA3 is associated with the hydrolysis of triglyceride in the liver, and the PNPLA3 rs738409 polymorphism is associated with the loss of the protein hydrolysis function and hepatic triglyceride accumulation. ${ }^{13}$ We recently showed that the PNPLA3 rs 738409 genotypes are associated with the risk of NAFLD in a Japanese general population, especially in normal-weight subjects, ${ }^{14}$ suggesting that the association between the PNPLA3 genotype and susceptibility to NAFLD is potentially influenced by the subjects' background. However, the effect of physical activity on the association between the PNPLA3 rs738409 polymorphism and the development of NAFLD remains unclear.

The objective of this exploratory longitudinal study among Japanese elderly subjects was to investigate the effect of physical activity on the risk for NAFLD while also paying attention to the PNPLA3 genotype.

\section{Materials and Methods}

A retrospective longitudinal analysis was conducted with 352 Japanese subjects recruited from among the participants in a health screening program conducted at the Japanese Red Cross Kumamoto Health Care Center between January 2006 and April 2012. Subjects with a habitual alcohol intake (consuming more than $30 \mathrm{~g} / \mathrm{day}$ of alcohol among men and $20 \mathrm{~g} /$ day among women) and/or with positive serological tests for hepatitis $\mathrm{B}$ and $\mathrm{C}$ viruses were excluded. The protocol of our study was approved by the ethics committee of the Faculty of Life Sciences, Kumamoto University, and the Japanese Red Cross Kumamoto Hospital Health Care Center. All subjects provided their written informed consent prior to participating in the study.

Clinical information was recorded at each follow-up visit. Generally, these visits were yearly; however, not all participants visited the center annually. The diagnosis of fatty liver disease was obtained by hepatic ultrasonography conducted by a radiographer. A medical doctor then reviewed the images to evaluate the accuracy and reproducibility of the diagnosis. When the subjects were diagnosed with NAFLD at baseline or during the observation period, they were grouped into the subjects with NAFLD at each follow-up visit after that time point to evaluate the incidence of NAFLD development using the data from various numbers of measurement points among the study subjects. The cumulative prevalence rate of NAFLD was used as a dependent variable in construction of the model. Physical activity was self-reported annually according to a detailed questionnaire regarding the type and frequency of physical activity. In addition, we collected the information on dietary habits at the end of observation period according to a questionnaire. The information was confirmed by face-toface interviews with medical staff members using a structured questionnaire. Each type of physical activity was assigned a metabolic equivalent (MET), which expresses the energy cost of physical activity; one MET was defined as the amount of oxygen consumed while a person was sitting quietly (e.g. METs of walking, swimming, hill climbing, jogging and cycling are 4.3, 6.0, 6.5, 7.5 and 8.0, respectively). ${ }^{15}$ We then calculated the "METS*T" value by METs $\times$ time for physical activity (hour/week) every year and time-average METS*T throughout the observation period for each subject (e.g. METS*T $=5: 10 \mathrm{mins} /$ day of walking; METS*T $=30: 1 \mathrm{hrs} /$ day of walking). The guidelines of the Korean Association for the Study of the Liver (KASL) for the management of NAFLD recommend performing exercise at a moderate intensity $(50 \%$ $70 \%$ of maximal heart rate) for at least 30 mins twice a week, which corresponds to 5 METS*T per week. ${ }^{16}$ Furthermore, the guidelines of the European Association for the Study of the Liver (EASL), European Association for the Study of Diabetes (EASD) and European Association for the Study of Obesity (EASO) for the management of NAFLD also recommend performing 150-200 min/week of moderate intensity 
aerobic physical activity, which is equivalent to 21 METS*T per week. ${ }^{17}$ Therefore, the subjects whose time-average METS*T was $<5$ were defined as the sedentary METS*T group, those with METS*T of 5 to $<21$ were the low METs*T group, and those with METS*T $\geq 21$ were the high METS*T group. The subjects were also grouped according to the METS*T tertiles (lowest group, METS*T <18.96; middle group, METS*T 18.96-42.13; highest group, METs*T >42.13).

Genomic DNA was extracted from whole blood using a DNA purification kit (FlexiGene DNA kit; Qiagen, Hilden, Germany). The PNPLA3 rs738409 (I148M or c. $444 \mathrm{C}>\mathrm{G}$ ) genotypes were determined using a real-time TaqMan allelic discrimination assay (Applied Biosystems, Foster City, CA, USA) according to the manufacturer's protocol (assay no. C_7241_10).

A one-way analysis of variance and Fisher's exact test were used for comparisons of continuous and categorical variables, respectively. In our previous study, we found that the risk of NAFLD showed tended to increase in the PNPLA3 rs738409 C/G and $\mathrm{G} / \mathrm{G}$ genotypes, with the risk in normal weight subjects with the $\mathrm{C} / \mathrm{G}$ genotype being approximately half that in normal weight subjects with the $\mathrm{G} / \mathrm{G}$ genotype. ${ }^{14}$ We therefore employed an additive genetic model to assess the genetic effects of PNPLA3 genotypes on the association between the risk of NALFD and physical activity. The longitudinal association of the PNPLA3 genotype and physical activity with the cumulative prevalence of NAFLD and the values of the body mass index (BMI) and waist circumstance were analyzed using a logistic regression analysis or multiple regression analysis based on generalized estimating equations, which allows for the analysis of repeated measurements when observations are missing. ${ }^{18,19}$ In these analyses, the interaction of PNPLA3 genotypes and physical activity was also investigated. These associations were calculated as the odds ratio (OR) and 95\% confidence intervals (CIs) for the risk of NAFLD and as the partial regression coefficient (B) and standard error (SE) for the BMI and waist circumstance. Based on the NAFLD prevalence and PNPLA3 genotype frequencies determined in our previous study, ${ }^{14}$ the total number of study subjects was estimated to be sufficient for detecting a $40 \%$ difference in the prevalence of NAFLD due to differences in physical activity with a power of 0.80 (a-level of 0.05 ). Moreover, we performed a sensitivity analysis to validate the ORs for the risk of NAFLD observed in the original data set. The replicated dataset for this analysis were generated from
10,000 randomly sampled subjects from original data set with replacement and stratified according to the study population to ensure a representative study population distribution using the individual as the sampling unit. The same statistical procedure as described above were used. A P value $<0.05$ was considered to be statistically significant.

\section{Results}

The mean follow-up duration was $4.8 \pm 1.4$ years. The frequencies of the PNPLA3 C/C, C/G and $\mathrm{G} / \mathrm{G}$ genotypes were $27.8 \%, 55.1 \%$ and $17.0 \%$, respectively. The observed PNPLA3 genotype frequency distribution was consistent with the Hardy-Weinberg equilibrium $(\mathrm{P}>0.05)$. The demographic characteristics at baseline did not differ markedly among the PNPLA3 genotypes (Table 1).

At the baseline, $53(15.1 \%)$ subjects in the overall population had NAFLD. The prevalence of NAFLD was higher (in order) in the PNPLA3 C/G, G/G, C/C genotypes (Table 1). The changes in the cumulative prevalence rate of NAFLD during observation period are shown in Supplementary Figures S1 and $\mathrm{S} 2$. The longitudinal cumulative prevalence of NAFLD was higher in the subjects with the PNPLA3 $\mathrm{C} / \mathrm{G}$ or $\mathrm{G} / \mathrm{G}$ genotypes than in those with the $\mathrm{C} / \mathrm{C}$ genotype (adjusted $\mathrm{OR}$ [95\% CI]: 2.66 [1.14-6.23], $\mathrm{P}<0.05 ; 2.02$ [1.01-4.06], $\mathrm{P}<0.05$, respectively), and the risks were consistent with previous reports. ${ }^{11}$ The longitudinal cumulative prevalence of NAFLD in the high METS*T and low METS*T groups tended to be lower in comparison to the sedentary METS*T group (adjusted ORs [95\% CIs]: 0.45 [0.18-1.14], P =0.091 and 0.59 [0.22-1.60], $\mathrm{P}=0.301)$.

An interactive effect of the PNPLA3 genotypes and EX levels on the cumulative prevalence of NAFLD was observed ( $\mathrm{P}=0.010)$. Among the PNPLA3 G/G genotype carriers, the $\mathrm{BMI}$ and waist circumstance were lower in the low METS*T and high METS*T groups than in sedentary METS*T group, whereas there were no significant associations of METS*T with the BMI or waist circumstance among the PNPLA3 C/C or $\mathrm{C} / \mathrm{G}$ genotypes carriers (Table 2 ). The cumulative prevalence of NAFLD was lower in the high and low METS*T groups than in the sedentary METS*T group among the PNPLA3 G/G genotype carriers and was equivalent to that among the $P N P L A 3 \mathrm{C} / \mathrm{C}$ or $\mathrm{C} / \mathrm{G}$ genotype carriers, in whom the risk of NAFLD did not differ among three METS*T groups (Figure 1 and Table 2). Furthermore, the logistic models of our sensitivity analysis also showed that the reducing effects of physical activity on the longitudinal prevalence of NAFLD were more pronounced in the PNPLA3 
Table I The Clinical Characteristics of the Study Subjects Stratified by the PNPLA3 Genotypes at Baseline

\begin{tabular}{|c|c|c|c|c|}
\hline & \multicolumn{3}{|c|}{ PNPLA3 Genotype } & \multirow[b]{2}{*}{$\mathbf{P}$} \\
\hline & $C C(n=98)$ & CG $(n=194)$ & GG $(n=60)$ & \\
\hline Female (\%) & $35(35.7)$ & $84(43.3)$ & $26(43.3)$ & 0.443 \\
\hline Age (years) & $67.9 \pm 5.6$ & $68.3 \pm 5.7$ & $67.7 \pm 5.7$ & 0.674 \\
\hline BMI $\left(\mathrm{kg} / \mathrm{m}^{2}\right)$ & $22.7 \pm 2.9$ & $22.5 \pm 2.7$ & $23.2 \pm 3.2$ & 0.231 \\
\hline Waist circumstance $(\mathrm{cm})$ & $82.0 \pm 7.0$ & $82.8 \pm 8.3$ & $84.2 \pm 8.2$ & 0.242 \\
\hline $\mathrm{HbAlc}(\%)$ & $5.81 \pm 0.67$ & $5.87 \pm 0.65$ & $5.77 \pm 0.51$ & 0.538 \\
\hline Fasting blood glucose (mg/dL) & $96(80-201)$ & $97(73-236)$ & $96(79-125)$ & 0.420 \\
\hline Systolic BP (mmHg) & $122.9 \pm 16.2$ & $122.7 \pm 18.2$ & $122.4 \pm 15.5$ & 0.983 \\
\hline Diastolic BP (mmHg) & $71.7 \pm 11.8$ & $71.5 \pm 10.7$ & $71.9 \pm 10.0$ & 0.974 \\
\hline HDL-C (mg/dL) & $68.8 \pm 17.1$ & $68.9 \pm 16.9$ & $68.6 \pm 15.3$ & 0.993 \\
\hline LDL-C (mg/dL) & $121.6 \pm 26.7$ & $124.6 \pm 27.4$ & $123.6 \pm 27.7$ & 0.676 \\
\hline TG (mg/dL) & $91.5(34-310)$ & 91 (34-272) & $94(42-264)$ & 0.991 \\
\hline AST (IU/L) & $23.7 \pm 5.0$ & $24.6 \pm 7.6$ & $24.9 \pm 6.8$ & 0.506 \\
\hline ALT (IU/L) & $21.0 \pm 7.2$ & $21.7 \pm 10.8$ & $22.6 \pm 11.4$ & 0.643 \\
\hline GGT (IU/L) & $22.5(10-135)$ & $22(7-259)$ & $25(10-174)$ & 0.402 \\
\hline NAFLD (\%) & $9(9.2)$ & $36(18.6)$ & $8(13.3)$ & 0.095 \\
\hline Diabetes (\%) & $13(13.3)$ & $23(11.9)$ & $9(15.0)$ & 0.743 \\
\hline Hypertension (\%) & $45(45.9)$ & $96(49.5)$ & $26(43.3)$ & 0.669 \\
\hline Dyslipidemia (\%) & $51(52.0)$ & $113(58.2)$ & $30(50.0)$ & 0.406 \\
\hline Ever smoking (\%) & $47(48.0)$ & $84(43.3)$ & $25(4 I .7)$ & 0.674 \\
\hline \multicolumn{5}{|l|}{ METS*T } \\
\hline Sedentary (\%) & $7(7.1)$ & II (5.7) & $5(8.3)$ & 0.142 \\
\hline Low (\%) & $22(22.4)$ & $72(37.1)$ & $18(30.0)$ & \\
\hline High (\%) & $69(70.4)$ & III (57.2) & 37 (61.7) & \\
\hline
\end{tabular}

Notes: The data are presented as the mean \pm standard deviation, median (range) or number of subjects (\%). METS*T, metabolic equivalent $\times$ time for physical activity (hour/week). Abbreviations: PNPLA3, patatin-like phospholipase domain containing protein 3; BMI, body mass index; BP, blood pressure; HDL-C, high-density lipoprotein cholesterol; LDL-C, low-density lipoprotein cholesterol, TG, triglyceride; AST, aspartate aminotransferase; ALT, alanine aminotransferase; GGT, gamma-glutamyl transferase; NAFLD, non-alcoholic fatty liver disease.

Table 2 The Effects of the Physical Activity Level on the Longitudinal Values of the BMI and Waist Circumstance and Longitudinal Prevalence of NAFLD Stratified by the PNPLA3 Genotypes

\begin{tabular}{|c|c|c|c|c|c|c|c|c|c|c|c|c|c|c|}
\hline \multirow{2}{*}{$\begin{array}{l}\text { PNPLA3 } \\
\text { Genotype }\end{array}$} & \multirow[t]{2}{*}{ METS*T } & \multirow[t]{2}{*}{$\mathbf{N}$} & \multicolumn{3}{|l|}{$\mathbf{B M I}^{\mathbf{a}}$} & \multicolumn{3}{|c|}{ Waist Circumstance $^{a}$} & \multicolumn{3}{|c|}{ NAFLD: Model I ${ }^{a}$} & \multicolumn{3}{|c|}{ NAFLD: Model $2^{b}$} \\
\hline & & & B & SE & $\mathbf{P}$ & B & SE & $\mathbf{P}$ & OR & $95 \% \mathrm{Cl}$ & $\mathbf{P}$ & OR & $95 \% \mathrm{Cl}$ & $\mathbf{P}$ \\
\hline \multirow[t]{3}{*}{$\mathrm{C} / \mathrm{C}$} & Sedentary & 7 & 0 & & & 0 & & & I & & & I & & \\
\hline & Low & 22 & 0.14 & 1.18 & 0.91 & -0.09 & 2.81 & 0.97 & 3.44 & $0.32-36.6$ & 0.31 & 8.40 & $0.52-137.1$ & 0.14 \\
\hline & High & 69 & -1.35 & 1.02 & 0.19 & -4.83 & 2.41 & $<0.05$ & 0.38 & $0.05-3.26$ & 0.38 & 1.31 & $0.13-12.92$ & 0.82 \\
\hline \multirow[t]{3}{*}{$\mathrm{C} / \mathrm{G}$} & Sedentary & 11 & 0 & & & 0 & & & I & & & I & & \\
\hline & Low & 72 & -1.60 & 1.45 & 0.27 & -6.86 & 3.73 & 0.07 & 0.42 & $0.11-1.63$ & 0.21 & 0.69 & $0.14-3.48$ & 0.65 \\
\hline & High & 111 & -1.55 & 1.43 & 0.28 & -6.66 & 3.67 & 0.07 & 0.53 & $0.14-1.95$ & 0.34 & 0.97 & $0.22-4.35$ & 0.97 \\
\hline \multirow[t]{3}{*}{ G/G } & Sedentary & 5 & 0 & & & 0 & & & 1 & & & I & & \\
\hline & Low & 18 & -4.28 & 1.73 & $<0.05$ & -9.49 & 3.73 & $<0.05$ & 0.13 & $0.02-0.69$ & $<0.05$ & 0.19 & $0.03-|.5|$ & 0.12 \\
\hline & High & 37 & -5.21 & 1.62 & $<0.01$ & -12.92 & 3.38 & $<0.001$ & 0.22 & $0.04-1.08$ & 0.06 & 0.44 & $0.06-3.30$ & 0.42 \\
\hline
\end{tabular}

Notes: ${ }^{\mathrm{a}} \mathrm{B}$ and $\mathrm{ORs}$ were adjusted by age, gender, smoking status, type 2 diabetes, dyslipidemia, hypertension. ${ }^{\mathrm{b}}$ ORs were adjusted by waist circumstance, age, gender, smoking status, type 2 diabetes, dyslipidemia, hypertension. METS*T, metabolic equivalent $\times$ time for physical activity (hour/week).

Abbreviations: NAFLD, non-alcoholic fatty liver disease; PNPLA3, patatin-like phospholipase domain containing protein 3; B, unstandardized partial regression coefficient; $\mathrm{SE}$, standard error; OR, odds ratio; $\mathrm{Cl}$, confidence interval. 

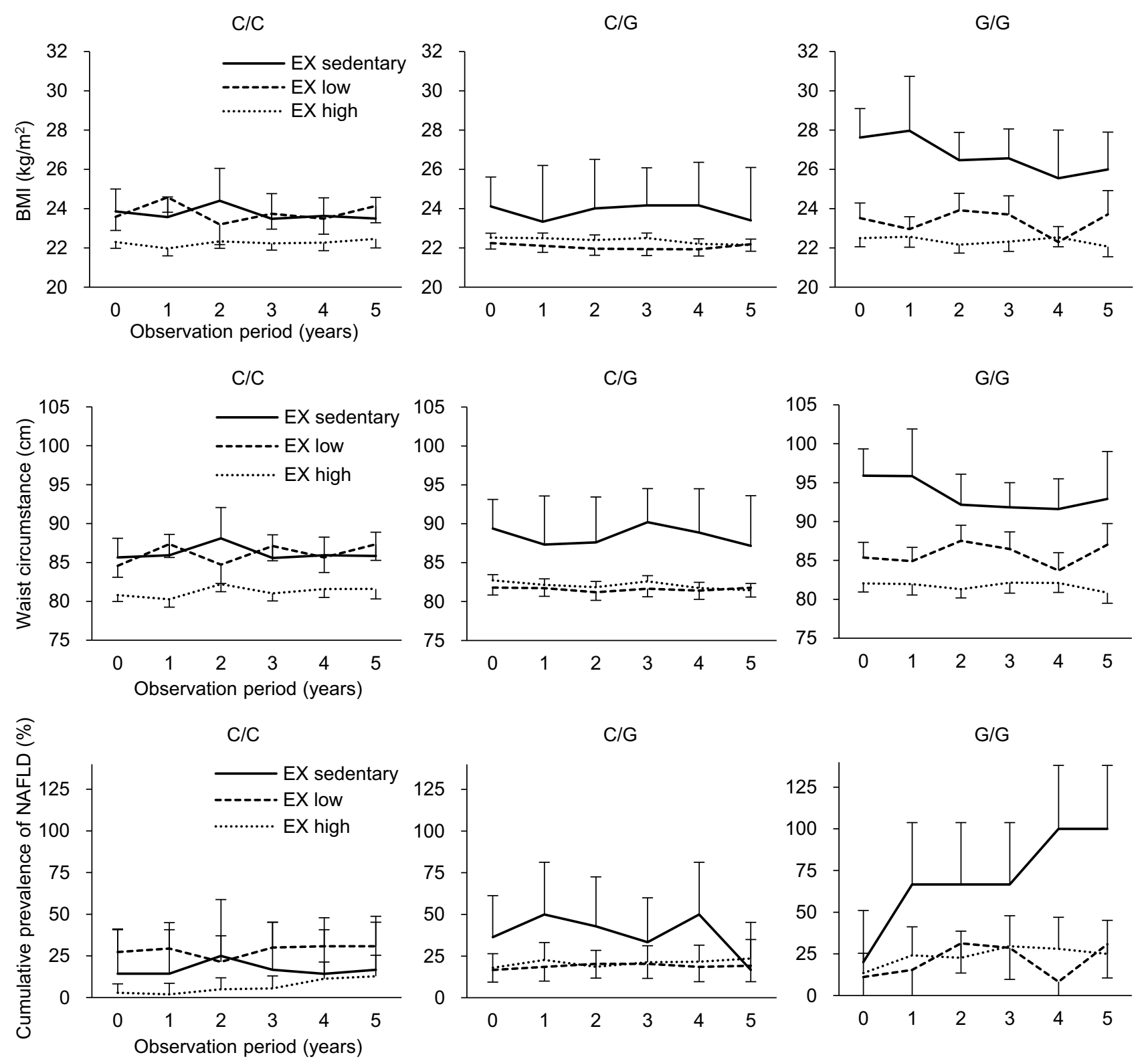

Figure I The association of physical activity with the longitudinal changes in the mean values of the BMI and waist circumstance and the cumulative prevalence of NAFLD separately in the subjects with PNPLA3 rs738409 C/C $(n=98), C / G(n=194)$ and $G / G(n=60)$ genotypes.

Notes: The bars represent the standard errors in the graphs regarding the BMI and waist circumstance or $95 \%$ confidence intervals in the graphs regarding the cumulative prevalence of NAFLD. METS*T, metabolic equivalent $\times$ time for physical activity (hour/week).

Abbreviations: BMI, body mass index; NAFLD, non-alcoholic fatty liver disease; PNPLA3, patatin-like phospholipase domain containing protein 3; EX, exercise.

$\mathrm{G} / \mathrm{G}$ genotype carriers than in the $\mathrm{C} / \mathrm{C}$ or $\mathrm{C} / \mathrm{G}$ genotypes carriers among the 10,000 randomly sampled subjects from the original data set (Supplementary Table S1). Therefore, we confirmed that the overall fitness of our NAFLD models seems to be good. We also analyzed the effect of the combination of the PNPLA3 genotypes with the METS*T values or METS*T tertiles on the cumulative prevalence of NAFLD. The METS*T values were not associated with the longitudinal cumulative prevalence of NAFLD (adjusted B [SE]: -0.04
[0.043], $\mathrm{P}=0.319)$. The METS*T tertiles were not also associated with the longitudinal cumulative prevalence of NAFLD, and the adjusted ORs [95\% CIs] in the highest group (METS*T >42.13) and the middle group (METS*T 18.96-42.13 ) vs the lowest group (METS*T of $<18.96$ ) were 0.56 [0.27-1.14] $(\mathrm{P}=0.110)$ and $0.85[0.43-1.68](\mathrm{P}=0.646)$, respectively. The interactive effects of the PNPLA3 genotype with the METS*T value or METS*T tertile were not observed in the logistic regression models for NAFLD ( $\mathrm{P}=0.889$ and 
0.336, respectively). Furthermore, the METS*T values and METS*T tertiles did not exhibit the dose-dependent effect on the risk of NAFLD and were not significantly associated with the cumulative prevalence of NAFLD regardless of the PNPLA3 genotypes (Supplementary Tables S2 and S3). In order to clarify whether or not dietary habits affected the physical activity in the study subjects, we analyzed the relationships between dietary habits and METS*T levels (Supplementary Table S4). No relationships between dietary habits and METS*T levels were observed (Supplementary Table S4).

\section{Discussion}

This is the first study to suggest that the PNPLA3 rs 738409 genotype may be associated with the inverse correlations of physical activity with the BMI, waist circumstance and NAFLD prevalence in a population of elderly Japanese subjects. Since the PNPLA3 G/G genotype increases the risk of NAFLD, ${ }^{11}$ physical activity as part of lifestyle modification (even if the METS*T is low) may be important for efficient and effective NAFLD prevention among these carriers.

A recent randomized controlled trial on a lifestyle modification program (i.e. conducting dietary and exercise consultations to increase energy expenditure and reduce caloric intake) conducted among NAFLD patients showed that the PNPLA3 rs738409 $\mathrm{G}$ allele was associated with a greater reduction in intrahepatic triglyceride levels, body weight and the waist-tohip ratio in the intervention group than the control group. ${ }^{20}$ Furthermore, a study regarding a weight-loss program conducted in obese children showed that PNPLA3 G/G genotype carriers achieved a greater reduction in the blood alanine aminotransferase level and waist-to-hip ratio than carriers of other genotypes. ${ }^{21}$ In another previous study, although the PNPLA3 G allele was strongly associated with the liver fat content and the risk of NAFLD, the insulin sensitivity was higher in the $\mathrm{G}$ allele carriers than in the $\mathrm{C} / \mathrm{C}$ genotype carriers among obese subjects. ${ }^{22}$ Taken together, routine physical activity (i.e. high or low METS*T) could be an effective intervention for preventing NAFLD, especially in PNPLA3 $\mathrm{G} / \mathrm{G}$ genotype carriers, possibly through these individuals being more sensitive to insulin than other genotype carriers. However, no significant effect of physical activity on the risk of NAFLD was observed in the PNPLA3 G/G genotype carriers after adjusting for the waist circumference (Table 2), suggesting that the decrease in the waist circumference by physical activity may be due to the beneficial effect of physical activity on the risk of NAFLD. However, the sensitivity analysis using the 10,000 randomly sampled subjects from the original data set showed a significant effect of physical activity on the risk of NAFLD in the PNPLA3 G/G genotype carriers even after adjusting for the waist circumference (Supplementary Table S1); these discrepant findings might be due to the small sample size in the original data set.

It should be noted that a number of behaviors are related to the weight status ${ }^{23,24}$ as well as the prevalence of NAFLD, ${ }^{9}$ and the relationship between physical activity and other behaviors (e.g. sedentary behaviors, poor sleep duration, unhealthy diet) and its influence on weight gain is complex. ${ }^{23,24}$ For example, an increase in sedentary behavior may be involved in the development of NAFLD independent of physical activity and should be considered when introducing lifestyle intervention. ${ }^{9}$ Cassidy et al showed that the incidence of low levels of physical activity, high television viewing and poor sleep duration is greater in obese or overweight adults than in normal or underweight adults, and these lifestyle behaviors are clustered in those individuals. ${ }^{23}$ In the present study, we found no relationships between dietary habits and METS*T levels (Supplementary Table S4). However, the information on dietary habits was brief and collected at the end of the observation period only. In addition, detailed information (e.g. precise carbohydrate, protein, and lipid intake) could not be obtained. Further studies are needed to develop appropriate PNPLA3 genotype-based lifestyle interventions incorporating subjects' behaviors other than their physical activity in order to prevent NAFLD development in elderly individuals.

Although NAFLD is closely related to weight gain and obesity, ${ }^{1}$ there is increasing interest in normal-weight NAFLD, which is commonly found in Asia. ${ }^{3,25}$ In Japan, normal-weight subjects account for $50 \%$ of the total NAFLD population, and $15.2 \%$ of the normal-weight population are diagnosed with NAFLD. ${ }^{3}$ Normal-weight individuals with NAFLD are more likely to have insulin resistance, type 2 diabetes and cardiovascular diseases than those without NAFLD, ${ }^{3,4}$ and they appear to have different characteristics compared to overweight or obese patients with NAFLD, such as a different distribution of visceral adipose tissue, recent increase in body weight, intake of a high-cholesterol diet and a characteristic genetic background. ${ }^{25}$ Our recent longitudinal study conducted in a Japanese general population showed that the PNPLA3 rs738409 genotypes are associated with the risk of NAFLD, especially in normal-weight subjects. ${ }^{14}$ Therefore, we speculate that physical activity may be important for NAFLD prevention among elderly individuals with 
the PNPLA3 G/G genotype, even if they have a normal weight status.

We also analyzed the combined effects of the PNPLA3 genotype with the METS*T values and METS*T tertiles on the cumulative prevalence of NAFLD in order to increase the number of subjects in each group. However, no interactive effects of the PNPLA3 genotype with the METS*T values or METS*T tertiles were seen. Furthermore, the METS*T values and METS*T tertiles did not exhibit the dosedependent effect on the risk of NAFLD in regardless of the PNPLA3 genotypes (Supplementary Tables $\mathrm{S} 2$ and $\mathrm{S} 3$ ). Regarding METS*T tertiles, it is possible that the relationship between physical activity and NAFLD could not be accurately analyzed because the METS*T value in the lowest group had a wider range ( 0 to 18.92). The results of this study suggest that the threshold METS*T level at which an improvement of NAFLD can be obtained is low (METS*T of $\geq 5$ ); thus, there was no relationship between physical activity and NAFLD in the lowest group (METS*T $\leq 18.92$ ).

Based on the NAFLD prevalence and PNPLA3 genotype frequencies in our previous study, ${ }^{14}$ we estimated a total number of study subjects of $\geq 350$ to be sufficient for detecting a $40 \%$ difference in the prevalence of NAFLD due to differences in physical activity with a power of 0.80 (a-level of 0.05). Conversely, the present study was unable to detect statistical significance of differences at less than $40 \%$, and a larger sample size would be required to elucidate the lack of any significant relationship between the cumulative prevalence of NAFLD and physical activity (e.g. the relationships among the carriers of the PNPLA3 $\mathrm{C} / \mathrm{C}$ or $\mathrm{C} / \mathrm{G}$ genotypes). Furthermore, although we estimated the total number of subjects necessary before starting this study, this was a preliminary study, and the number of study subjects was small, which was a major limitation of the present study. Therefore, we performed internal validation analyses using a replicated data set consisting of 10,000 random samples from original data sets and confirmed the reducing effect of physical activity on the cumulative prevalence of NAFLD, especially in the PNPLA3 G/G genotype (Supplementary Table S1). However, since the analyses were conducted with the subjects obtained from the original data, further investigations in larger and more varied populations are needed in order to verify the preliminary findings.

In this study of health screening program participants, since not all participants visited the center annually, when the subjects were diagnosed with NAFLD at baseline or during the observation period, they were grouped into the subjects with NAFLD at each follow-up visit after that time point to evaluate the incidence of NAFLD development using data obtained from study subjects at various numbers of measurement points. Moreover, the number of subjects suffering from NAFLD at the baseline was 53 and the subjects with NAFLD at baseline were not excluded to avoid limiting the datasets unnecessarily. Thus, we used a logistic regression analysis, but not a Cox-hazards regression analysis, which is generally used to analyze cumulative incidence, based on generalized estimating equations, which allows for the analysis of repeated measurements with missing observations. ${ }^{18,19}$ For model construction, the cumulative prevalence rate of NAFLD at each observation period was used as a dependent variable. On the other hand, 53 subjects were suffering from NAFLD at baseline, and 15 of these subjects achieved remission during the observation period. However, we could not evaluate the factors associated with the remission of NAFLD due to the small sample size, which was a limitation of this study.

In the present study, reducing effects of physical activity (low or high METS*Ts) on the cumulative prevalence of NAFLD were not observed in the PNPLA3 $\mathrm{C} / \mathrm{C}$ or $\mathrm{C} / \mathrm{G}$ genotype carriers (Table 2), suggesting that high and low METS*T may not be associated with a lower risk of NAFLD than sedentary METS*T in these genotype carriers. However, in our sensitivity analysis, the reducing effects of physical activity on the risk of NAFLD were observed in the PNPLA3 C/C or $\mathrm{C} / \mathrm{G}$ genotype carriers among the 10,000 randomly sampled subjects from the original data set (Supplementary Table S1), although the magnitude of the association between physical activity and the risk of NAFLD tended to be less in PNPLA3 C/C and C/G genotype carriers than in the G/G genotype carriers (Supplementary Table S1). The lack of a beneficial effect of physical activity on the risk of NAFLD among the PNPLA3 $\mathrm{C} / \mathrm{C}$ and $\mathrm{C} / \mathrm{G}$ genotype carriers may be due to the small sample size of our original data set. However, among PNPLA3 C/C genotype carriers, a decrease in the risk of NAFLD was observed only at high METS*T (i.e. $\geq 21$ METS*T) in the sensitivity analyses (Supplementary Table S1). In addition, the tertiles of METS*T levels suggest that the middle and highest METS*T (i.e. >18.96 METS*T) had a protective effect against the risk of NAFLD in the PNPLA3 C/C genotype carriers (Supplementary Table S2). We speculate that the amount of physical activity required to see a beneficial effect on the risk of NAFLD may differ among PNPLA3 genotypes, and $P N P L A 3 \mathrm{C} / \mathrm{C}$ genotype carriers may require more 
physical activity to achieve a beneficial effect on the risk of NAFLD than G/G genotype carriers. Further investigations will be needed in order to examine whether or not the beneficial effects of physical activity on the risk of NAFLD are more pronounced in the PNPLA3 G/G genotype carriers than in others.

The prevalence of NAFLD was lower in PNPLA3 G/G genotype carriers than in the $\mathrm{C} / \mathrm{G}$ genotype carriers at the baseline, although previous studies have revealed that PNPLA3 G/G genotype carriers have a higher risk of NAFLD than $\mathrm{C} / \mathrm{G}$ genotype carriers. ${ }^{3,11,12}$ However, in the second and subsequent years, the prevalence tended to be higher in PNPLA3 G/G genotype carriers than in $\mathrm{C} / \mathrm{G}$ genotype carriers (Supplementary Figure S2). Of note, the study subjects were participants in a Japanese health screening program and might have had a high level of health literacy. In addition, they were relatively old (mean age: $67.5 \pm 6.0$ years old at baseline and $72.9 \pm$ 6.1 years old at endpoint). Therefore, carriers of the highrisk genotype (i.e. PNPLA3 G/G genotype) may take extra precautions to stay healthy due to prior experiences with NAFLD. Furthermore, the sample size of this study was relatively small, especially when the subjects were stratified by the PNPLA3 genotypes. The higher prevalence of NAFLD in the $\mathrm{C} / \mathrm{G}$ genotype might be due to selection bias and/or a small sample size, although the details are unclear.

Since this study retrospectively collected clinical information from elderly participants attending a health screening program, we used hepatic ultrasonography to diagnose FLD, with the findings reviewed by medical specialists; this approach is known to have a sensitivity of $64 \%$ and a specificity of $97 \%$ in detecting fatty liver. ${ }^{26}$ Further investigations are thus needed to verify the findings using a liver biopsy for the diagnosis of FLD. In addition, the data on the physical activity in this study could not be thoroughly validated. Specifically, physical activity levels in the study participants have only been measured by faceto-face interviews with medical staff members using a structured questionnaire, which might have lacked reliability because of the recall and social desirability bias. ${ }^{9}$

\section{Conclusion}

Despite the above-mentioned limitations, this pilot study provides the useful information for generating further intervention program to prevent development of NAFLD based on the physical activity and PNPLA3 genotype in the general elderly population. The current preliminary results suggest that the PNPLA3 rs738409 genotype may be associated with the beneficial effects of physical activity on the risk of NAFLD among elderly Japanese subjects. Although elderly populations may be physically limited to some degree, impressing the importance of physical activity, even as little as $30 \mathrm{~min} /$ day of walking (METS*T $=14$, average METS*T among the low METS*T group), upon PNPLA3 G/G genotype carriers may potentially help prevent NAFLD. However, since the present study did not use thoroughly validated physical activity data or other lifestyle information, our results warrant further comprehensive investigations incorporating the well-validated physical activity data and detailed information about other behaviors besides physical activity in larger and more diverse populations, including other age groups and ethnic groups, to verify the findings.

\section{Acknowledgments}

The authors thank all of the study participants and the staff of the Japanese Red Cross Kumamoto Health Care Center. The work was supported by grants-in-aid for scientific research from the Ministry of Education, Culture, Sports, Science and Technology, Japan (Grant Numbers: 17K15510 and 16K08406) and the Japan Research Foundation for Clinical Pharmacology.

\section{Disclosure}

The authors declare that they have no conflicts of interest.

\section{References}

1. Drew L. Fighting the fatty liver. Nature. 2017;550:S102-S103. doi:10.1038/550S102a

2. Sarwar R, Pierce N, Koppe S. Obesity and nonalcoholic fatty liver disease: current perspectives. Diabetes Metab Syndr Obes. 2018;11:533-542. doi:10.2147/DMSO

3. Seto WK, Yuen MF. Nonalcoholic fatty liver disease in asia: emerging perspectives. J Gastroenterol. 2017;52:164-174. doi:10.1007/s00535016-1264-3

4. Yoshitaka H, Hamaguchi M, Kojima T, et al. Nonoverweight nonalcoholic fatty liver disease and incident cardiovascular disease: a post hoc analysis of a cohort study. Medicine. 2017;96:e6712. doi:10.1097/ MD.0000000000006712

5. Borel AL, Nazare JA, Smith J, et al. Visceral, subcutaneous abdominal adiposity and liver fat content distribution in normal glucose tolerance, impaired fasting glucose and/or impaired glucose tolerance. Int $J$ Obes (Lond). 2015;39:495-501. doi:10.1038/ijo.2014.163

6. Dhana K, Berghout MA, Peeters A, et al. Obesity in older adults and life expectancy with and without cardiovascular disease. Int $J$ Obes (Lond). 2016;40:1535-1540. doi:10.1038/ijo.2016.94

7. Gan L, Chitturi S, Farrell GC. Mechanisms and implications of age-related changes in the liver: nonalcoholic fatty liver disease in the elderly. Curr Gerontol Geriatr Res. 2011;2011:831536. doi:10.1155/2011/831536 
8. Miyake T, Kumagi T, Hirooka M, et al. Significance of exercise in nonalcoholic fatty liver disease in men: a community-based large cross-sectional study. J Gastroenterol. 2015;50:230-237. doi:10.1007/ s00535-014-0959-6

9. Romero-Gomez M, Zelber-Sagi S, Trenell M. Treatment of nafld with diet, physical activity and exercise. J Hepatol. 2017;67:829-846. doi:10.1016/j.jhep.2017.05.016

10. Hashida R, Kawaguchi T, Bekki M, et al. Aerobic vs. Resistance exercise in non-alcoholic fatty liver disease: a systematic review. $J$ Hepatol. 2017;66:142-152. doi:10.1016/j.jhep.2016.08.023

11. Zhang L, You W, Zhang H, et al. Pnpla3 polymorphisms (rs738409) and non-alcoholic fatty liver disease risk and related phenotypes: a meta-analysis. J Gastroenterol Hepatol. 2015;30:821-829. doi:10.1111/ jgh.2015.30.issue-5

12. Shen JH, Li YL, Li D, et al. The rs738409 (i148m) variant of the pnpla3 gene and cirrhosis: a meta-analysis. J Lipid Res. 2015;56:167-175.

13. Huang Y, Cohen JC, Hobbs HH. Expression and characterization of a pnpla3 protein isoform (i148m) associated with nonalcoholic fatty liver disease. J Biol Chem. 2011;286:37085-37093. doi:10.1074/jbc. M111.290114

14. Oniki K, Saruwatari J, Izuka T, et al. Influence of the pnpla3 rs738409 polymorphism on non-alcoholic fatty liver disease and renal function among normal weight subjects. PLoS One. 2015;10: e0132640. doi:10.1371/journal.pone.0132640

15. Jette M, Sidney K, Blumchen G. Metabolic equivalents (mets) in exercise testing, exercise prescription, and evaluation of functional capacity. Clin Cardiol. 1990;13:555-565. doi:10.1002/clc.v13:8

16. The Korean Association for the Study of the Liver. KASL clinical practice guidelines: management of nonalcoholic fatty liver disease. Clin Mol Hepatol. 2013;19:325-348. doi:10.3350/cmh.2013.19.4.325

17. European Association for the Study of the Liver, European Association for the Studyof Diabetes, European Association for the Study of Obesity. EASL-EASD-EASO clinical practice guidelines for the management of non-alcoholic fatty liver disease. J Hepatol. 2016;64:1388-1402. doi:10.1016/j.jhep.2015.11.004
18. Wang M, Kong L, Li Z, et al. Covariance estimators for generalized estimating equations (gee) in longitudinal analysis with small samples. Stat Med. 2016;35:1706-1721.

19. Salazar A, Ojeda B, Duenas M, et al. Simple generalized estimating equations (gees) and weighted generalized estimating equations (wgees) in longitudinal studies with dropouts: guidelines and implementation in r. Stat Med. 2016;35:3424-3448. doi:10.1002/ sim. 6947

20. Shen J, Wong GL, Chan HL, et al. Pnpla3 gene polymorphism and response to lifestyle modification in patients with nonalcoholic fatty liver disease. J Gastroenterol Hepatol. 2015;30:139-146. doi:10.1111/ jgh. 12656

21. Marzuillo P, Grandone A, Perrone L, et al. Weight loss allows the dissection of the interaction between abdominal fat and pnpla3 (adiponutrin) in the liver damage of obese children. $J$ Hepatol. 2013;59:1143-1144. doi:10.1016/j.jhep.2013.06.027

22. Kantartzis K, Peter A, Machicao F, et al. Dissociation between fatty liver and insulin resistance in humans carrying a variant of the patatin-like phospholipase 3 gene. Diabetes. 2009;58:2616-2623. doi:10.2337/db09-0279

23. Cassidy S, Chau JY, Catt M, et al. Low physical activity, high television viewing and poor sleep duration cluster in overweight and obese adults; a cross-sectional study of 398,984 participants from the uk biobank. Int J Behav Nutr Phys Act. 2017;14:57. doi:10.1186/s12966-017-0514-y

24. Lampure A, Castetbon K, Hanafi M, et al. Relative influence of socioeconomic, psychological and sensory characteristics, physical activity and diet on 5-year weight gain in french adults. Nutrients. 2017;9:1179. doi:10.3390/nu9111179

25. Liu CJ. Prevalence and risk factors for non-alcoholic fatty liver disease in asian people who are not obese. J Gastroenterol Hepatol. 2012;27:1555-1560. doi:10.1111/j.1440-1746.2012.07222.x

26. Smith BW, Adams LA. Nonalcoholic fatty liver disease and diabetes mellitus: pathogenesis and treatment. Nat Rev Endocrinol. 2011;7:456-465. doi:10.1038/nrendo.2011.72

Diabetes, Metabolic Syndrome and Obesity: Targets and Therapy

Dovepress

Publish your work in this journal

Diabetes, Metabolic Syndrome and Obesity: Targets and Therapy is an international, peer-reviewed open-access journal committed to the rapid publication of the latest laboratory and clinical findings in the fields of diabetes, metabolic syndrome and obesity research. Original research, review, case reports, hypothesis formation, expert opinion and commentaries are all considered for publication. The manuscript management system is completely online and includes a very quick and fair peer-review system, which is all easy to use. Visit http://www.dovepress.com/testimonials.php to read real quotes from published authors.

Submit your manuscript here: https://www.dovepress.com/diabetes-metabolic-syndrome-and-obesity-targets-and-therapy-journal 